\title{
Observed fearlessness and positive parenting interact to predict childhood callous-unemotional behaviors among low-income boys
}

\author{
Rebecca Waller, ${ }^{1}$ Daniel S. Shaw, ${ }^{2}$ and Luke W. Hyde ${ }^{3}$ \\ ${ }^{1}$ Department of Psychology, Department of Psychiatry, University of Michigan, Ann Arbor, MI; ${ }^{2}$ Department of \\ Psychology, University of Pittsburgh, Pittsburgh, PA; ${ }^{3}$ Department of Psychology, Center for Human Growth and \\ Development, Survey Research Center of the Institute for Social Research, University of Michigan, Ann Arbor, MI,
} USA

\begin{abstract}
Background: Callous-unemotional behaviors identify children at risk for severe and chronic antisocial behavior. Research is needed to establish pathways from temperament and parenting factors that give rise to callousunemotional behaviors, including interactions of positive versus harsh parenting with child fearlessness. Methods: Multimethod data, including parent reports and observations of parent and child behavior, were drawn from a prospective, longitudinal sample of low-income boys $(N=310)$ with assessments at 18,24 , and 42 months, and at ages 10-12 years old. Results: Parent-reported callous-unemotional, oppositional, and attention-deficit factors were separable at 42 months. Callous-unemotional behaviors at 42 months predicted callous-unemotional behaviors at ages 10-12, accounting for earlier oppositional and attention-deficit behaviors and self-reported child delinquency at ages 10-12. Observations of fearlessness at 24 months predicted callous-unemotional behaviors at 42 months, but only when parents exhibited low observed levels of positive parenting. The interaction of fearlessness and low positive parenting indirectly predicted callous-unemotional behaviors at 10-12 via callous-unemotional behaviors at 42 months. Conclusions: Early fearlessness interacts with low positive parenting to predict early callousunemotional behaviors, with lasting effects of this person-by-context interaction on callous-unemotional behaviors into late childhood. Keywords: Callous-unemotional; parenting; psychopathy; temperament.
\end{abstract}

\section{Introduction}

Childhood antisocial behavior, including aggression, theft, and substance use, represents a significant public health concern. It undermines children's socioemotional development and academic achievement, causes harm within families and schools, and presents significant financial cost to society (Scott, Knapp, Henderson, \& Maughan, 2001). A key challenge to research seeking to establish the origins of antisocial behavior is that many young children show normative behavior problems during the preschool period, including aggression or temper tantrums. However, while some of these children continue to experience problems, showing lifecourse antisocial behavior that persists into adolescence and adulthood, others naturally desist from early behavior problems (Campbell, Shaw, \& Gilliom, 2000). Thus, research has sought to determine why some children recover, but others show persistent antisocial behavior. This knowledge can lead to more effective targeting of preventative interventions.

\section{Callous-unemotional behaviors}

Although several child risk factors have been linked to risk for persistent antisocial behavior beginning

Conflict of interest statement: No conflicts declared. in early childhood, including low inhibitory control, fearlessness, and high negative emotionality (Campbell et al., 2000), one of the most consistently predictive factors is callous-unemotional behaviors (Frick, Ray, Thornton, \& Kahn, 2014; Waller, Shaw, Neiderhiser, et al., 2017). Measures of callousunemotional behaviors were developed as a downward extension of interpersonal/affective traits found in psychopathy (Frick et al., 2014), which predicts violent and nonviolent antisocial conduct among adults (Blair, Peschardt, Budhani, Mitchell, $\&$ Pine, 2006). In children, measures of callousunemotional behaviors assess low empathy, lack of guilt, and reduced emotional responsivity to others (Frick et al., 2014). Across late childhood and adolescence, callous-unemotional behaviors robustly predict future antisocial behavior (Frick et al., 2014). Recently, research has examined callous-unemotional behaviors during early childhood (i.e. ages 2-4) because this period is when the developmental foundations are laid for empathy and conscience (Kochanska, 1997) and when severe trajectories of antisocial behavior have their origins (Shaw, Gilliom, Ingoldsby, \& Nagin, 2003). Thus, research focused on toddlerhood, when children begin to show more opposition and aggression (Campbell et al., 2000), could improve our understanding of the development of callous-unemotional behaviors, and identify children at risk for persistent and severe behavior problems across development. 
To assess early callous-unemotional behaviors, studies have used items from common behavior rating scales (Willoughby, Waschbusch, Moore, \& Propper, 2011). Across five separate samples of 3 year olds (not including the present sample), this five-item measure of callous-unemotional behaviors has been shown to form a separate factor from a sixitem oppositional and six-item attention-deficit scale (e.g. Willoughby, Mills-Koonce, Gottfredson, \& Wagner, 2014; Willoughby et al., 2011). This measure of callous-unemotional behaviors uniquely predicts later behavior problems over and above early oppositional or attention-deficit behaviors (Waller, Shaw, Neiderhiser, et al., 2017; Willoughby et al., 2014). Moreover, as evidence of construct validity, in a separate sample of 3-year-old children recruited based on elevated risk for externalizing problems, callous-unemotional behaviors were correlated with lower empathy, guilt, and moral regulation (Waller, Hyde, Grabell, Alves, \& Olson, 2015). Finally, in another independent sample of 731 low-income children in a study of a preventative intervention, a similar measure of early callous-unemotional behaviors at age 3 predicted callous-unemotional behaviors at age 9.5 measured by a well-established and longer measure of the construct (Waller, Dishion, et al., 2016). Thus, across multiple samples, early callous-unemotional behaviors predict later behavior problems, are related concurrently to low empathy, and are related prospectively to a traditional measure of callous-unemotional behaviors. However, this final study is the only one to date to have examined the convergence of early measure callous-unemotional behaviors with callous-unemotional behaviors in late childhood. More longitudinal evidence is needed to establish that early childhood measures show construct validity relative to measures assessed in late childhood or adolescence. Thus, the first aim of the present study was to examine whether a five-item measure of callous-unemotional behaviors in early childhood predicted callous-unemotional behaviors in early adolescence.

\section{Fearlessness and callous-unemotional behaviors}

While callous-unemotional behaviors in early childhood have been shown to foreshadow behavior problems later in childhood, we know less about how callous-unemotional behaviors develop, particularly during very early childhood when life span trajectories of antisocial behavior have their origins and when individual differences in callous-unemotional behaviors emerge. Drawing on its origins in the psychopathy literature, callous-unemotional behaviors are hypothesized to emerge from a biologically conferred 'fearless' temperament. Fearlessness is central to the developmental models of psychopathy (Blair et al., 2006) and conscience (Kochanska, 1997), as it is thought to confer low arousal to others' distress and punishment, leading to reduced learning about the outcomes of harmful behavior, and increasing risk for callous-unemotional behaviors (Blair et al., 2006). Although prominent in theory, only two longitudinal studies have linked fearlessness in the first years of life to later callous-unemotional behaviors; one to callous-unemotional behaviors at age 13 in a longitudinal, population-based study (Barker, Oliver, Viding, Salekin, \& Maughan, 2011) and the other to callous-unemotional behaviors in early childhood within an adoption study that demonstrated the heritable nature of fearlessness (Waller, Trentacosta, et al., 2016).

\section{Person-by-context interactions}

In testing potential temperamental pathways, it is also important to consider that pathways from temperament to personality and behavior are modified by context, particularly parental caregiving (Kochanska, 1997). Harsh parenting is thought to interfere with children's ability to internalize rules and develop conscience. In contrast, mutually positive and responsive parenting is thought to facilitate children's ability to internalize parental messages of socialization or rules/norms and develop empathy (Kochanska, 1997; Waller et al., 2014). The independent effects of both harsh and positive parenting are thought to be important among fearless children who may be at greater risk for callous-unemotional behaviors (Kochanska, 1997). Indeed, both parental harshness and low warmth have been found to predict increases in callous-unemotional behaviors during early childhood (e.g. Mills-Koonce, Willoughby, Garrett-Peters, Wagner, \& Vernon-Feagans, 2016; Waller et al., 2014). Moreover, in recent work using an adoption design, child fearlessness was found to be inherited from biological mothers, but fearlessness only predicted early callous-unemotional behaviors in the context of low positive parenting by adoptive parents (Waller, Trentacosta, et al., 2016). These findings suggest that positive parenting is important in preventing pathways to callous-unemotional behaviors and behavior problems, particularly among young children showing a fearless temperament. However, this single study using an adoption design focused on children adopted into middle-class homes with few contextual stressors. Moreover, this prior study assessed callous-unemotional behaviors up to 27 months only and was not able to examine longer term consequences of early fearlessness and low positive parenting. Thus, more research is needed in higher risk samples with longer follow-up periods. In particular, research is needed to establish whether positive parenting interacts with fearlessness in the prediction of child callous-unemotional among typical 'biological families', particularly in those at elevated risk for antisocial behavior based on low socioeconomic and high-risk urban contexts. 
Moreover, measures of positive parenting are typically inversely correlated with measures of parental harshness. Few studies exist that have examined both positive and harsh parenting in relation to callous-unemotional behaviors or fearlessness in a single model, which makes it difficult to know whether parental harshness accounts for the effects reported for positive parenting in prior studies. Indeed, the influence of harsh versus positive parenting on callous-unemotional behaviors could be distinct after accounting for their correlation (for a systematic review, see Waller, Gardner, \& Hyde, 2013). For example, Pasalich and colleagues reported that the relationship between callous-unemotional behaviors and behavior problems was buffered by positive parenting during the preschool period, after accounting for harsh parenting (Pasalich, Dadds, Hawes, \& Brennan, 2011). Moreover, Barker et al. (2011) found that harsh parenting in early childhood exacerbated the effects of a fearless temperament on children's callous-unemotional behaviors at age 13, controlling for low parental warmth. Finally, MillsKoonce et al. (2016) found that observations of low parental sensitivity and harsh-intrusive parenting from ages 0-36 months both uniquely predicted higher callousness at age 6 . Together, these findings suggest that both parental harshness and positive parent-child interactions could be important for understanding developmental pathways to callousunemotional behaviors. Modeling their effects simultaneously could help to establish unique moderating effects of harsh and positive parenting practices on pathways from fearlessness to callous-unemotional behaviors in early childhood and subsequent callous-unemotional and antisocial behavior in later childhood.

\section{Current study}

We examined observed measures of early fearlessness and positive versus harsh parenting as predictors of callous-unemotional behaviors from early to late childhood in a sample of 310 low-income boys. First, as we had not previously examined early callous-unemotional behaviors in this study, we established that callous-unemotional behaviors factored separately from oppositional and attentiondeficit behaviors at 42 months (Willoughby et al., 2014). We hypothesized that early callous-unemotional behaviors at 42 months would uniquely predict callous-unemotional behaviors in late childhood at ages 10-12, taking into account attention-deficit and oppositional behaviors at 42 months and child self-reported delinquency at ages 10-12. Second, we hypothesized that observed fearlessness at 24 months would uniquely predict parent-reported callous-unemotional behaviors during the preschool period beyond overlap with oppositional and attention-deficit behaviors. Moreover, we hypothesized that child fearlessness at 24 months would interact with both maternal harsh and positive parenting to predict early callous-unemotional behaviors, such that high levels of maternal harshness and low positive parenting would independently exacerbate risk. Finally, we hypothesized indirect effects, such that fearlessness and parenting at 24 months would interact to predict callous-unemotional behaviors in late childhood via preschool-aged callous-unemotional behaviors. Because studies have found callous-unemotional behaviors to be highly heritable (Viding, Fontaine, \& McCrory, 2012) and studies have shown that the broader context of the family (e.g. low family income, neighborhood impoverishment) influence the development of callous-unemotional behaviors (Waller, Shaw, Forbes, \& Hyde, 2015), we examined study questions in a conservative model that controlled for maternal aggressive personality traits and low empathy (to model potential risky heritable effects), as well as family income and neighborhood impoverishment (to model risky contextual predictors)

\section{Methods Participants}

Participants are from the Pittsburgh Mother \& Child Project, a prospective longitudinal study of low-income families with multiinformant and observed measures of parent and child behavior (Shaw et al., 2003). In 1991 and 1992, 310 infant boys and their mothers were recruited from Women, Infants, and Children Nutrition Supplement Clinics in Allegheny County, Pennsylvania, when boys were between 6-17 months old. During recruitment, 53\% of mothers reported that they self-identified as European American, 36\% as African American, $5 \%$ as biracial, and $6 \%$ as other races. Two thirds of mothers in the sample had 12 years of education or less. The mean per capita income was $\$ 241$ per month, and mean Hollingshead socioeconomic status score was 24.5. Thus, many boys in this study were considered at elevated risk for antisocial behavior because of socioeconomic standing. Retention rates were high at follow-up assessments that were conducted almost annually from 18 months, with data available for $90 \%$ of participants at 42 months and $86 \%$ of participants at ages 10,11 , or 12 . Independent samples t-tests comparing participants who were lost versus retained showed that children not retained at 42 months not differ on any measures in the current study. Children lost versus retained at 10-12 years old also did not differ on any measures in the current study, with the exception of having lower scores for oppositional behaviors $(t=2.21, d f=277$, $p=.03)$ and callous-unemotional behaviors $(t=2.14, d f=277$, $p=.03)$ at 42 months compared to children who were retained.

\section{Procedure and ethical considerations}

For the current study, we used observational and questionnaire data from 2- to 3-hr visits collected in the home and/or lab at ages 1.5, 3.5, and ages 10, 11, and 12. At each assessment, parents consented to participate, completed questionnaires regarding sociodemographic characteristics, family issues, and child behavior, and engaged in structured and semistructured tasks for observational coding (Shaw et al., 2003). All assessments and measures were approved by the IRB of the University of Pittsburgh. 


\section{Measures}

Observed fearlessness (24 months). We assessed nonsocial behavioral inhibition (fearlessness-fearfulness) using a system based on Kochanska (1991) and adapted by Shaw et al. (2003). Ratings of fearlessness were assessed during a Scary Gorilla Task using two molecular and two global scales (Shaw et al., 2003). The molecular codes were (a) latency to approach the scary gorilla sounds and (b) time in proximity to mother. The global ratings were (a) distress and (b) approach/ avoidance. Distress was based on duration and intensity of facial and vocal expressions of fear/anxiety (e.g. crying; whimpering; distress). Ratings of approach/avoidance were based on the extent to which boys approached and investigated the cabinet. Global ratings were made on 4-point scales. Interrater reliability was $\geq .90$ based on $20 \%$ of cases coded by two raters To create a fearlessness construct, ratings were subjected to principal components analysis, which yielded one factor and accounted for $49 \%$ of the variance. In a prior study in the current sample, fearlessness predicted trajectories of aggressive and oppositional behavior from ages 2-8 (Shaw et al., 2003).

Observed harsh and positive parenting (24 months). We assessed harsh and positive dimensions of parenting at 24 months using two separate observational methods. To examine (1) harsh parenting, videotapes of a clean-up task using the Early Parenting Coding System (Winslow \& Shaw, 1995) were coded using two molecular ratings - verbal/ physical approval and critical statements - and three global ratings - hostility, warmth, and punitiveness. For molecular ratings, Cohen's kappa coefficients ranged from .79 to .83 . Principal components analysis of the two molecular and three global ratings yielded a single harsh parenting factor (Shaw et al., 2003). To examine (2) Positive parenting, we used the Home Observation for Measurement of the Environment (Caldwell \& Bradley, 1984), which assessed support and stimulation in the home via semistructured observation and interviews with parents. The eight-item acceptance subscale taps parent's responses to child misbehavior or distress $(\alpha=.67$; see Shaw et al., 2003 for more details).

\section{Early childhood callous-unemotional behaviors} (42 months). We assessed early callous-unemotional behaviors at 42 months using 17 items of the Achenbach System of Empirically Based Assessment (Achenbach \& Rescorla, 2000). We used a three-factor structure to parse heterogeneity in early behavior problems, including a five-item callous-unemotional, six-item oppositional, and six-item attention-deficit factors (see Willoughby et al., 2011, 2014). By measuring callous-unemotional behaviors within a 3-factor model, we could establish that pathways from fearlessness and parenting were specific to callous-unemotional behaviors. As we had not previously examined early callous-unemotional behaviors in this sample, we present results testing the 3 -factor model (Figure 1). We reran subsequent analyses controlling for callous-unemotional behaviors at 24 months to account for autoregressive effects; results were unchanged.

\section{Late childhood callous-unemotional behaviors}

(ages 10-12). To examine callous-unemotional behaviors in late childhood, we used a parent-reported measure of limited prosocial emotions derived in a prior study using the current sample (Waller, Shaw, Forbes, et al., 2015). The measure comprised four items of the callous-unemotional scale of the Antisocial Process Screening Device (APSD; Frick \& Hare, 2001; for example, 'feels guilty after wrongdoing') at ages 10 and 11, and 10 items of the Prosociality/Empathy scale of the Child and Adolescent Dispositions Scale (CADS; Lahey, Rathouz, Applegate, Tackett, \& Waldman, 2010) at age 12 (e.g. 'helps others when they get hurt'). The APSD and CADS scales were combined in a single latent factor to provide a robust measure of callous-unemotional behaviors across ages 10-12 (see Waller, Shaw, Forbes, et al., 2015).

Late childhood self-reported delinquency (ages $10-12)$. To confirm that the findings were specific to callous-unemotional behaviors and not broader antisocial behavior, we examined children's antisocial behavior using the Selfreport Delinquency Questionnaire (Elliott, Huizinga, \& Ageton, 1985), which assesses the frequency that children have engaged in 33 different types of antisocial, aggressive, and delinquent behavior, substance use, and related offenses in the last year $(\alpha=.79-.92$; Waller, Shaw, Forbes, et al., 2015).

Covariates. In addition to (1) Child race: $(0=$ European American; 1 = non-European American), we accounted for factors that could influence the parents' abilities to provide a nurturing environment and factors that could indicate heritable risk for callous-unemotional behaviors, including maternal personality traits or characteristics and contextual factors: (2) Parent low empathy (24 months) assessed via parent report on the Adolescent Parenting Inventory (Bavolek, Kline, McLaughlin, \& Publicover, 1979), a 32-item measure that assesses maternal characteristics and beliefs. The low empathy scale contains eight items assessing empathetic attitudes relating to parenting (e.g. 'sensitive parents spoil children'). The low empathy scale was used previously in this sample, exhibiting good internal consistency $(\alpha=.81)$ and predicted child callousunemotional behaviors at ages 10-12 (Waller, Shaw, Forbes, et al., 2015). (3) Parent aggressive personality (18 months) assessed via the 16-item Aggression subscale of the Personality Research Form (Jackson, 1989; for example, 'I fly into a rage if things don't go as planned'). Aggression scores showed modest acceptable internal reliability in a previous study in this sample $(\alpha=.63$; Waller, Shaw, Forbes, et al., 2015). (4) Neighborhood risk (18 months) assessed via geocoding of addresses using census data coded at the block group level (Vanderbilt-Adriance \& Shaw, 2008). (5) Family monthly income was ascertained during demographic interviews.

\section{Analytic strategy}

Aim 1: Three-factor model in early childhood and prediction of late childhood outcomes. We specified 17-items of the ASEABA at 42 months to load onto separate callous-unemotional, attention-deficit, and oppositional factors (Willoughby et al., 2011). We used confirmatory factor analysis in Mplus version 7.2 (Muthén \& Muthén, 2014) with mean and variance adjusted weighted least squares estimation (WLSMV) appropriate for ordinal items. Using corrected chisquare differences test, we compared the model fit for this three-factor model to single-factor and two-factor models. To examine construct and predictive validity, we tested pathways from callous-unemotional, attention-deficit, and oppositional behaviors at 42 months to callous-unemotional behaviors and self-reported delinquency at ages 10-12.

Aim 2: Effects of fearlessness and parenting on three-factor model in early childhood and pathways to callous-unemotional behaviors in late childhood. We examined main and interactive effects of observed fearlessness at 24 months, and observed harsh versus positive parenting at 24 months on callous-unemotional, attention-deficit, and oppositional behaviors at age 42 months within the three-factor model using WLSMV estimation. We controlled for the effects of child race, neighborhood risk, and family income, as well as maternal aggressive personality traits and low empathy. In a final path model, we tested whether 


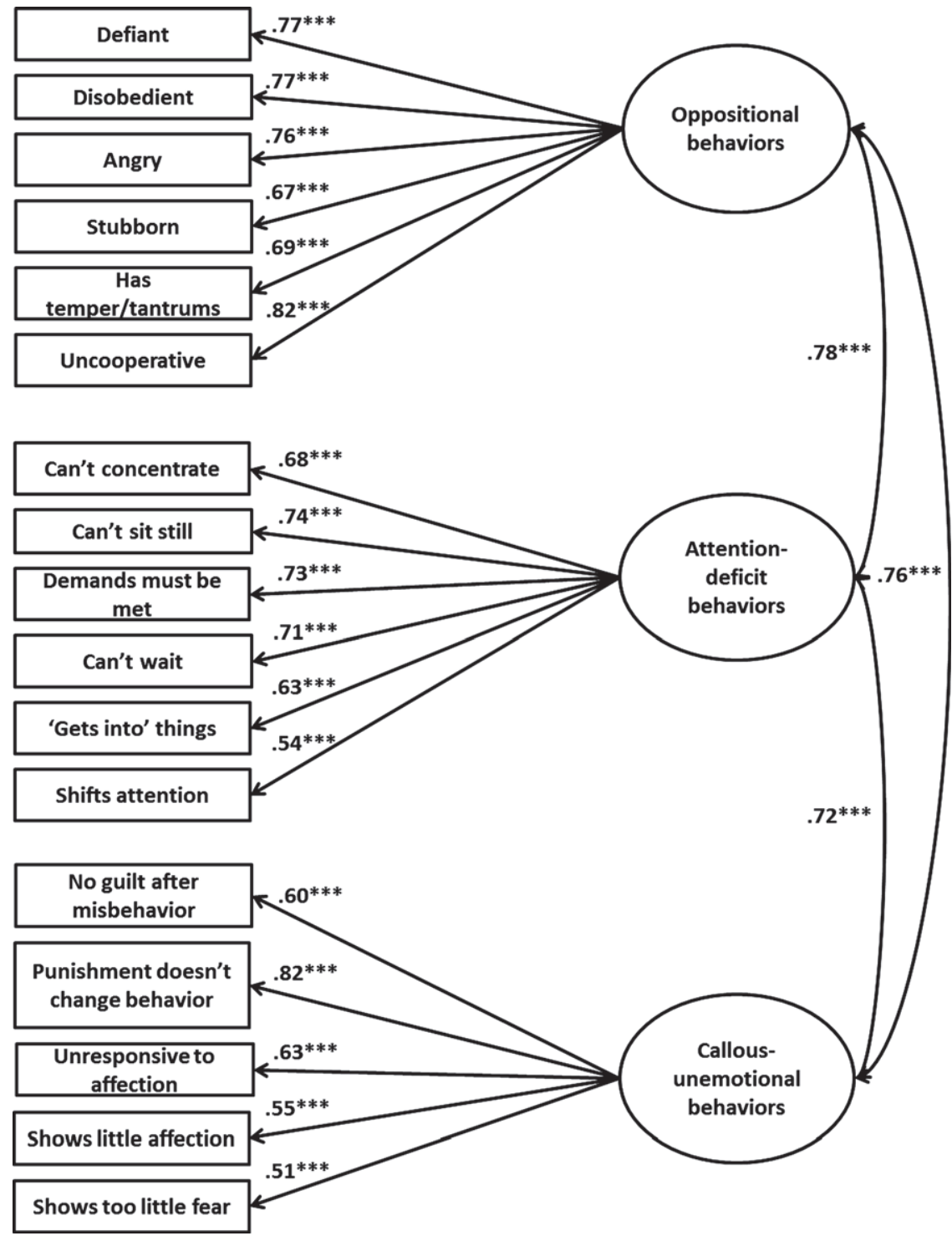

$* * * p<.001$. Fit for three-factor model was significantly better than one-and two-factor models (Table S3).

Figure 1 Best-fitting three-factor model with separable factors for oppositional, attention-deficit, and callous-unemotional behaviors at 42 months. ${ }^{* *} p<.001$. Fit for three-factor model was significantly better than one- and two-factor models (Table S3)

parenting-fearlessness interactions predicted callous-unemotional behaviors at ages $10-12$ (i.e. either directly or indirectly via callous-unemotional behaviors at 42 months; Figure S3). ${ }^{1}$

\section{Results}

Descriptive statistics are presented in Table 1; bivariate correlations between study variables are presented in Table S1.

\section{Aim 1: Predictive validity of early childhood callous- unemotional behaviors}

Consistent with prior studies in other samples, a three-factor model showed good fit to the data and significantly better fit than one- or two-factor models in the current sample (Figure 1; see Table S3 for model comparisons). Callous-unemotional behaviors at 42 months uniquely predicted higher callous- unemotional behaviors at ages $10-12 \quad(B=.62$, $S E=.21, \beta=.62, p<.01)$, accounting for oppositional and attention-deficit behaviors and controlling for child race, family income, and neighborhood risk. There was a trend-level association between oppositional behaviors at 42 months and higher selfreported delinquency at ages $10-12 \quad(B=.97$, $S E=.52, \beta=.26, p<.10$; Table S2). Thus, callousunemotional behaviors formed a separate dimension from other early problem behaviors, and demonstrated unique construct and predictive validity.

Aim 2: Effects of fearlessness and parenting on three-factor model in early childhood and prediction of late childhood callous-unemotional behaviors

Within a structural equation model examining the unique effects of parenting, child fearlessness, and 
their interactive terms (Figure S2), there was no main effect of child fearlessness at 24 months on callous-unemotional behaviors at 42 months (Table 2), although observed positive parenting at 24 months predicted both attention-deficit and callous-unemotional behaviors at 42 months. ${ }^{2}$ Moreover, there was a significant interaction between fearlessness and positive parenting in the prediction of child callous-unemotional behaviors at 42 months. We probed this interaction at low $(1 S D<M)$ versus high positive parenting (1 $S D>M$; Aiken, West, \& Reno, 1991). Child fearlessness predicted higher callous-unemotional behaviors at 42 months among children who experienced low $(B=.31, S E=.09$, $\beta=.43, p<.001)$, but not high positive parenting at 24 months $(B=-.11, S E=.16, \beta=-.09, p>.50$; Figure 2). ${ }^{3}$ There were no unique main or interactive effects of harsh parenting, although low parent empathy uniquely predicted higher callous-unemotional behaviors at 42 months, and parent aggressive traits predicted higher scores for all three factors (Table 2). ${ }^{4}$

In a final model, we added pathways to callousunemotional behaviors and self-reported delinquency at ages 10-12. Consistent with hypotheses, there was a significant indirect pathway from the interaction between positive parenting and fearlessness at 24 months on callous-unemotional behaviors at ages $10-12$ via callous-unemotional behaviors at 42 months and a significant indirect pathway from positive parenting to callous-unemotional behaviors at ages 10-12 via callous-unemotional behaviors at 42 months (Figure 3).

\section{Discussion}

We report evidence establishing the developmental origins of callous-unemotional behaviors in late childhood beginning with an interaction between toddler-age parenting and fearlessness via the development of preschool callous-unemotional behaviors.

Table 1 Descriptive statistics

\begin{tabular}{lcc}
\hline & $N$ & $M(S D)$ \\
\hline Covariates (18-24 months) & & \\
1. Family income & 307 & $1,044.90(642.25)$ \\
2. Neighborhood risk & 300 & $0.39(1.18)$ \\
3. Parent low empathy & 299 & $17.01(4.78)$ \\
4. Parent aggressive traits & 301 & $6.94(2.92)$ \\
Main effects (24 months) & & \\
5. Child observed fearlessness & 220 & $-0.01(1.00)$ \\
$\quad$ 6. Observed harsh parenting & 287 & $0.07(3.44)$ \\
7. Observed positive parenting & 290 & $4.97(1.94)$ \\
Child outcomes (42 months) & & \\
$\quad$ 8. Callous-unemotional behaviors & 279 & $0.47(.34)$ \\
9. Attention-deficit behavior & 279 & $1.00(.42)$ \\
10. Oppositional behaviors & 279 & $0.79(.45)$ \\
Child outcomes (10-12 years) & & \\
11. Callous-unemotional & 181 & $0.00(1.00)$ \\
$\quad$ behaviors (10-12 years) & & \\
12. Self-reported & 267 & $3.52(3.36)$ \\
$\quad$ delinquency (10-12 years) & & \\
\hline
\end{tabular}

These results build on existing literature providing a model across childhood where early callous-unemotional behaviors arise from the person-by-context interaction of low positive parenting and child fearlessness, predicting callous-unemotional behaviors 8 years later. Fearlessness was a specific temperamental precursor to callous-unemotional behaviors, although only when boys experienced low positive parenting. Findings were robust within multivariate models that accounted for other early behavior problems, harsh parenting, and the influence of contextual risk and maternal personality traits, strengthening the conclusions that can be drawn.

A burgeoning area of research has begun to examine the origins of early callous-unemotional behaviors. In support of contextual and parenting influences being important to the development of callous-unemotional behaviors, we found that low positive parenting at 24 months predicted later callous-unemotional and attention-deficit behaviors at age 42 months. Moreover, positive parenting at 24 months predicted callous-unemotional behaviors in early adolescence, via callous-unemotional behavior at 42 months. However, this main effect of low positive parenting was qualified by an interaction between positive parenting and fearlessness in predicting callous-unemotional behaviors. These findings are consistent with the concept of multifinality; the idea that two children who both experience low positive parenting ultimately present with different phenotypes (i.e. attention-deficit vs. callous-unemotional behaviors), dependent on unique aspects of child temperament (i.e. fearlessness). The moderation findings are similar to a previous study in a different sample that found positive parenting mitigated the effects of earlier fearlessness on callousunemotional behaviors within an adoption study (Waller, Trentacosta, et al., 2016). As this previous study used an adoption design, we can be more confident that parenting effects are nonheritable. Moreover, the effect of low positive parenting on the development of callous-unemotional behaviors complements findings from other samples that have linked lower positive parenting to callous-unemotional behaviors during early childhood (e.g. MillsKoonce et al., 2016; Waller et al., 2014). Thus, highly positive parenting, over and above the effects of harshness, appears uniquely important for preventing the development of callous-unemotional behaviors, specifically by attenuating and buffering risky temperamental pathways to early childhood callous-unemotional behaviors (Kochanska, 1997; Waller, Trentacosta, et al., 2016). These findings are paramount for informing preventative interventions that could identify children via temperament risk for callous-unemotional behaviors and attempt to buffer this risk by increasing positive parenting.

Interestingly, we found no main effect of fearlessness on later callous-unemotional behaviors either in bivariate or multivariate models (i.e. link was only 
Table 2 Effects of early fearlessness and parenting on child callous-unemotional, attention-deficit, and oppositional behaviors

\begin{tabular}{|c|c|c|c|c|c|c|}
\hline \multirow[b]{3}{*}{ All paths examined simultaneously } & \multicolumn{6}{|c|}{ Within three-factor model (Figure 1) } \\
\hline & \multicolumn{2}{|c|}{$\begin{array}{l}\text { Attention-deficit } \\
\text { behaviors }\end{array}$} & \multicolumn{2}{|c|}{ Oppositional behaviors } & \multicolumn{2}{|c|}{$\begin{array}{c}\text { Callous-unemotional } \\
\text { behaviors }\end{array}$} \\
\hline & $B(S E)$ & $\beta$ & $B(S E)$ & $\beta$ & $B(\mathrm{~S} E)$ & $\beta$ \\
\hline \multicolumn{7}{|l|}{ Covariates (18 or 24 months) } \\
\hline Child race & $.01(.14)$ & .003 & $.13(.14)$ & .06 & $-.03(.16)$ & -.01 \\
\hline Family income & $.004(.12)$ & .002 & $-.14(.11)$ & -.08 & $-.04(.13)$ & -.02 \\
\hline Neighborhood risk & $.04(.08)$ & .04 & $.04(.07)$ & .04 & $.06(.08)$ & .05 \\
\hline Maternal low empathy & $.001(.01)$ & -.002 & $-.03(.02)$ & -.11 & $.04(.02)$ & $.14^{*}$ \\
\hline Maternal aggressive traits & $.06(.03)$ & $.17^{*}$ & $.10(.03)$ & $.27^{* * *}$ & $.08(.03)$ & $.21^{* *}$ \\
\hline \multicolumn{7}{|l|}{ Main effects (24 months) } \\
\hline Child fearlessness & $.06(.08)$ & .06 & $.06(.08)$ & .05 & $-.06(.09)$ & -.06 \\
\hline Harsh parenting & $-.01(.02)$ & -.02 & $-.02(.02)$ & -.04 & $-.04(.02)$ & -.11 \\
\hline Positive parenting & $-.09(.04)$ & $-.16^{*}$ & $-.07(.04)$ & $-.12^{\dagger}$ & $-.10(.04)$ & $-.17^{*}$ \\
\hline \multicolumn{7}{|l|}{ Interactions ( 24 months) } \\
\hline Child fearlessness $\times$ Harsh parenting & $.04(.02)$ & $.11^{\dagger}$ & $.01(.02)$ & $.03(.07)$ & $-.01(.04)$ & -.02 \\
\hline Child fearlessness $\times$ Positive parenting & $-.04(.04)$ & -.07 & $-.06(.04)$ & $-.12(.08)$ & $-.16(.06)$ & $-.28 * *$ \\
\hline
\end{tabular}

${ }^{\dagger} p<.10 ;{ }^{*} p<.05 ; * * p<.01 ; * * * p<.001$.

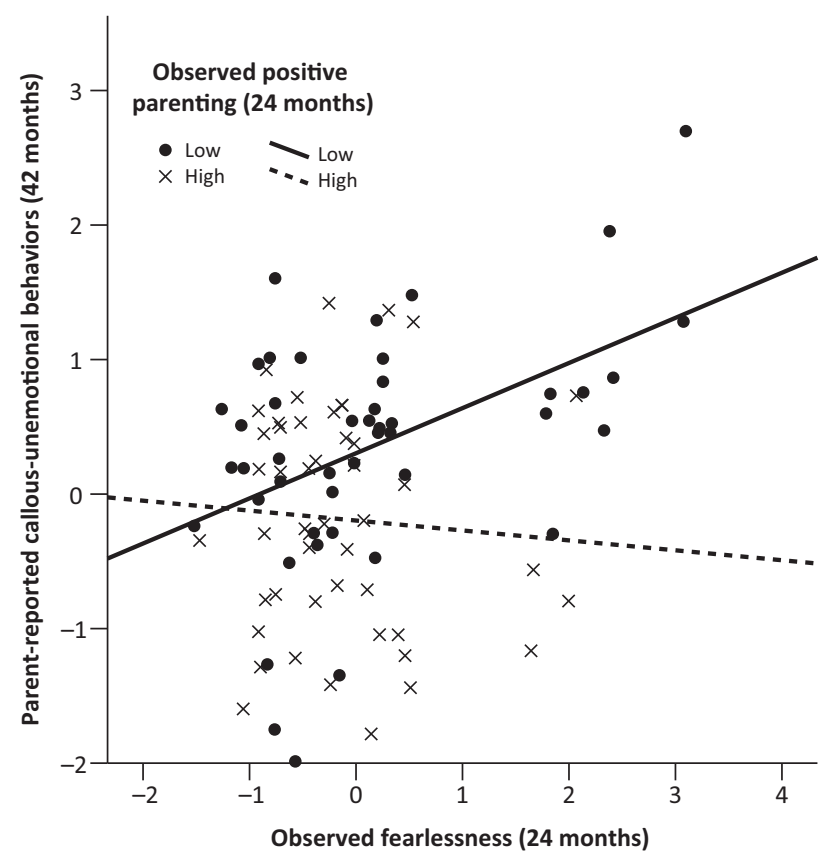

Figure 2 Fearlessness predicts later callous-unemotional behaviors only among boys who experience low positive parenting

apparent at low positive parenting). This null finding contradicts our hypothesis and the abovementioned adoption study that used a similar observational paradigm and the same measure of callous-unemotional behaviors at a comparable age (Waller, Trentacosta, et al., 2016). One explanation arises from sample differences. In the earlier study, the link between fearlessness and callous-unemotional behaviors was established among children placed with adoptive families who are not representative of typical families, having significantly higher income and fewer contextual risk factors for antisocial behavior (Waller, Trentacosta, et al., 2016). In contrast, in the current sample, we examined boys only and low-income biological families living in impoverished neighborhoods. Nevertheless, in a prior study in the current sample, highly fearless boys did exhibit severe and stable behavior problems from 2-8 years old, although callous-unemotional behaviors were not assessed (Shaw et al., 2003). Thus, in this sample, fearlessness may be less effective at differentiating boys at specific risk for callous-unemotional behaviors, but may represent a general risk marker for antisocial behavior in a context defined by greater risk or may only be a risk factor for callous-unemotional behaviors in the context of low positive parenting.

Finally, beyond establishing an interaction between low positive parenting and fearlessness in predicting early callous-unemotional behaviors, the results provide support for the separability of items tapping the callous-unemotional construct from other markers of behavior problems at age 3. The current study adds to existing literature by demonstrating unique convergence of callous-unemotional behaviors at age 3.5 with a measure of callous-unemotional behaviors at ages 10-12. Only one study to date has established a link between measures of callous-unemotional behaviors across childhood. Specifically, in a sample of low-income families involved in a preventative intervention, callous-unemotional behaviors at age 3 uniquely predicted callous-unemotional behaviors at age 9.5, taking into account informant perceptions within a multitrait multimethod approach (Waller, Dishion, et al., 2016). Drawing across the findings of these two studies, there is growing evidence that this type of measure of early callous-unemotional behaviors exhibits homotypic continuity across childhood.

Although our study had strengths, including a prospective longitudinal design over 10 years, observational measurement, and stringent multivariate models, we note several limitations. First, we included only boys from low-income families living in an urban setting. These findings may not 


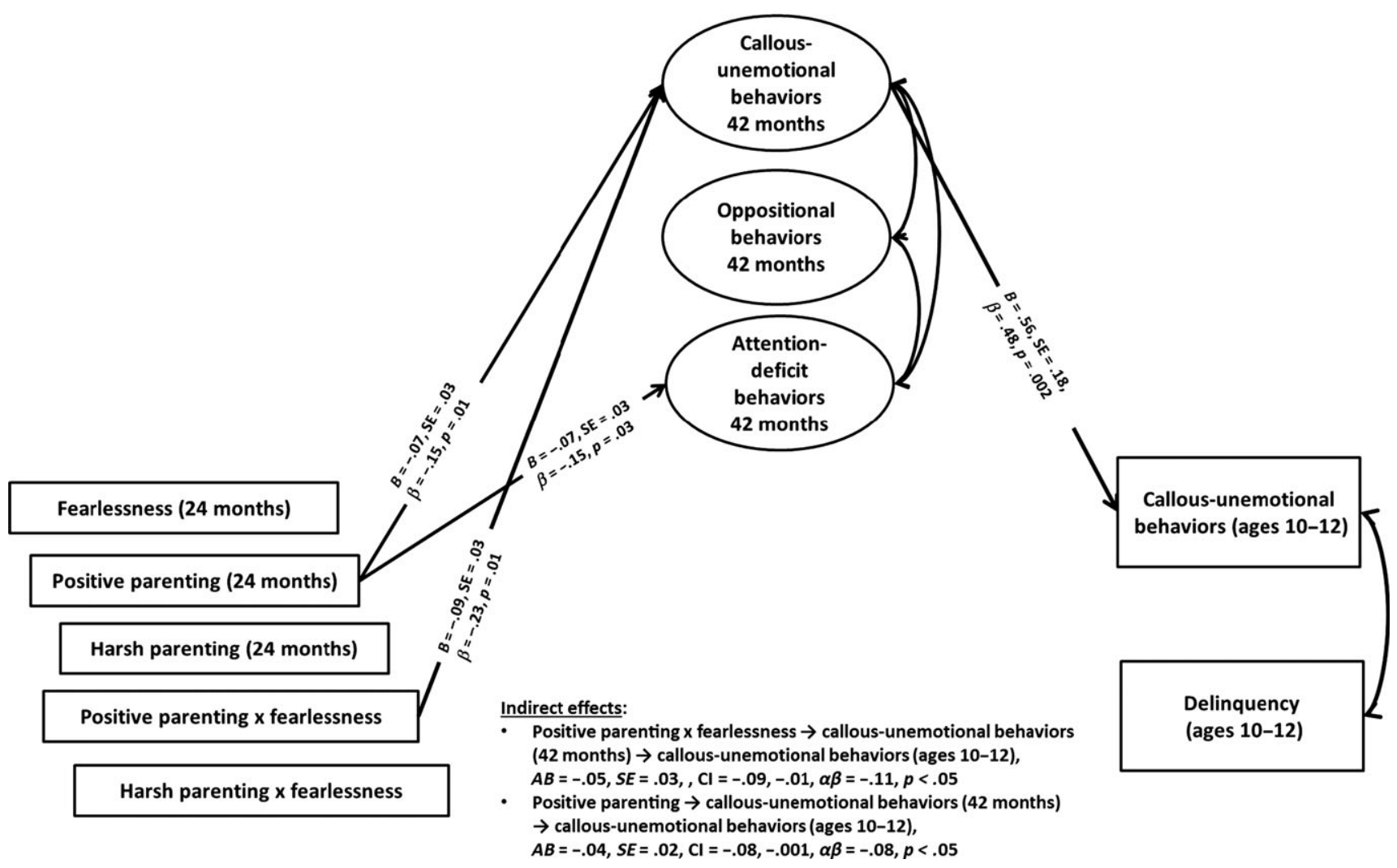

Figure 3 Positive parenting and fearlessness interact to predict late childhood callous-unemotional behaviors specifically via early childhood callous-unemotional behaviors. Note. For ease of interpretation, only significant pathways shown. See Figure S3 for all paths modeled, including effects of covariates: maternal aggression, maternal low empathy, family income, neighborhood impoverishment, and child race

generalize to girls or higher socioeconomic or nonurban settings. Second, although we accounted for personality characteristics of mothers, models were not examined within a genetically informed design and findings could reflect shared genetic vulnerability. Moreover, although we accounted for maternal traits, recent studies have shown that father's personality traits are related to boys' callous-unemotional behaviors, which were not measured here (Dadds et al., 2014). Finally, although we used a well-established measure of fearlessness indexed by a lack of nonsocial behavioral inhibition (i.e. to scary gorilla sounds), it has yet to be established the extent to which this construct taps into 'social fearlessness', relevant to theories that callous-unemotional behaviors develop because children are unresponsive following parental efforts to punish their transgressions. Future studies could test this question by examining whether low fear or reduced autonomic arousal to nonsocial threats (e.g. scary sounds/toys) predicts children's subsequent fearlessness to social/interpersonal threats (i.e. to parental punishment, reinforcement of the rules, or displeasure at children's transgressions), and in turn, whether this social fearlessness predicts the development of callous-unemotional behaviors.

In sum, we provide evidence of a temperament pathway from fearlessness to early callous-unemotional behaviors in the context of low positive parenting. This temperament-context interaction had lasting consequences, indirectly predicting children's callous-unemotional behaviors during the transition to adolescence via earlier callous-unemotional behaviors. This work provides further evidence that low positive parenting is related to increased risk of children developing callous-unemotional behaviors, particularly among children with a fearless temperament. The results have implications for developmental models of callousunemotional behaviors as a risk marker for severe antisocial behavior, and preventative interventions that seek to mitigate these risky pathways.

\section{Supporting information}

Additional Supporting Information may be found in the online version of this article:

Table S1. Bivariate correlations between study variables.

Table S2. Unique effects of early child callous-unemotional, attention-deficit, and oppositional behaviors.

Table s3. Synopsis of Confirmatory Factor Analysis models testing 17 items of the ASEBA and comparing model fit via the DIFFTEST procedure.

Table S4. Effects of early fearlessness and parenting on child callous-unemotional, attention-deficit, and oppositional behaviors.

Figure S1. Model examining unique effects of oppositional, attention-deficit, and callous-unemotional 
behaviors at 42 months on later callous-unemotional behaviors.

Figure s2. Model testing main and interactive pathways from child fearlessness, and positive and harsh parenting to separate oppositional, attentiondeficit, and callous-unemotional behavior factors. Figure S3. Model examined to test indirect and direct pathways from positive versus harsh parenting.

\section{Acknowledgements}

This research was supported by grants to D.S.S. from the National Institute of Mental Health (MH50907 and MH01666) and the National Institute on Drug Abuse
(DA25630 and DA26222). R.W. was supported in her efforts by a NIAAA T32 Fellowship in the Addiction Center, Department of Psychiatry, University of Michigan (2T32AA007477-24A1). The authors are grateful to the staff of the Pitt Mother $\&$ Child Project for their years of service and to our study families for making the research possible. The authors have declared that they have no competing or potential conflicts of interest.

\section{Correspondence}

Luke W. Hyde, Department of Psychology, University of Michigan, 530 Church St., Ann Arbor, MI 48109, USA;

Email: lukehyde@umich.edu

\section{Key points}

- Callous-unemotional behaviors increase risk for severe and persistent antisocial behavior.

- Little research has examined temperamental and parenting risk factors for callous-unemotional behaviors in early childhood.

- This prospective longitudinal study established that child fearlessness in toddlerhood uniquely predicted callous-unemotional behaviors in early childhood, but only when parents showed low positive parenting.

- This fearlessness $\times$ low positive parenting interaction also predicted callous-unemotional behaviors in late childhood via callous-unemotional behaviors in early childhood.

- Prevention programs focused on increasing positive parenting could mitigate risk for early callousunemotional behaviors and subsequent antisocial behavior, particularly among children with risky fearless temperaments.

\section{Notes}

1. For ease of computation based on the model complexity, we used extracted factor scores for callous-unemotional, oppositional, and attentiondeficit behaviors and maximum likelihood estimation with robust standard errors (MLR). We resorted to a model using MLR with extracted factor scores because the model using WLSMV and the full threefactor measurement structure for the callous-unemotional, oppositional, and attention-deficit scales would not converge, likely because of the combination of categorical and continuous mediating and outcome variables in a complex model. However, the extracted factor scores for the callous-unemotional, oppositional, and attention-deficit scales were based on a measurement model using WLSMV appropriate for use with ordinal items (Aim 1; Figure 1) and thus approximate the ideal model while lowering computational complexity.

2. The positive parenting-fearlessness interaction term continued to uniquely predict callous-unemotional behaviors in a model where we removed the 'shows low fear' item as an indicator of the callousunemotional latent factor. The estimates from this additional analysis are presented in Table S4 and suggest that the significant effects of the interaction cannot be explained by overlap between fearlessness as a predictor (i.e. parenting $\times$ fearlessness) and fearlessness as an indicator of the criterion variable (i.e. the callous-unemotional behavior construct). 3. Figure 2 indicates the existence of a child with extremely high callous-unemotional behaviors (3 $S D>$ mean). A sensitivity analysis was computed where this outlier was removed, resulting in the same pattern of findings (i.e. the interaction term in the continuous analysis was significant and the pattern for the simple slope analysis was similar), thus increasing confidence in the robustness of the findings.

4. The pattern of findings for the positive parentingfearlessness interaction was unchanged in a model when harsh parenting, maternal aggression and low empathy, and other covariates were not included that is, findings were not contingent on the presence of these covariates. Thus, findings from the more stringent model and a model without covariates converged.

\section{References}

Achenbach, T.M., \& Rescorla, L. (2000). ASEBA preschool forms \& profiles: An integrated system of multi-informant assessment. Burlington, VT: Department of Psychiatry, University of Vermont.

Aiken, L.S., West, S.G., \& Reno, R.R. (1991). Multiple regression: Testing and interpreting interactions. Newbury Park, CA: Sage. 
Barker, E.D., Oliver, B.R., Viding, E., Salekin, R.T., \& Maughan, B. (2011). The impact of prenatal maternal risk, fearless temperament and early parenting on adolescent callous-unemotional traits: A 14 year longitudinal investigation. Journal of Child Psychology and Psychiatry, 52, 878888.

Bavolek, S.J., Kline, D.F., McLaughlin, J.A., \& Publicover, P.R. (1979). Primary prevention of child abuse and neglect: Identification of high-risk adolescents. Child Abuse and Neglect, 3, 1071-1080.

Blair, R.J.R., Peschardt, K., Budhani, S., Mitchell, D., \& Pine, D. (2006). The development of psychopathy. Journal of Child Psychology and Psychiatry, 47, 262-276.

Caldwell, B.M., \& Bradley, R.H. (1984). Home observation for measurement of the environment. Little Rock, AR: University of Arkansas at Little Rock.

Campbell, S.B., Shaw, D.S., \& Gilliom, M. (2000). Early externalizing behavior problems: Toddlers and preschoolers at risk for later maladjustment. Development and Psychopathology, 12, 467-488.

Dadds, M.R., Allen, J.L., McGregor, K., Woolgar, M., Viding, E., \& Scott, S. (2014). Callous-unemotional traits in children and mechanisms of impaired eye contact during expressions of love: A treatment target? Journal of Child Psychology and Psychiatry, 55, 771-780.

Elliott, D.S., Huizinga, D., \& Ageton, S.S. (1985). Explaining delinquency and drug use. Beverly Hills, CA: Sage.

Frick, P.J., \& Hare, R.D. (2001). Antisocial process screening device: APSD. Toronto, Canada: Multi-Health Systems.

Frick, P.J., Ray, J.V., Thornton, L.C., \& Kahn, R.E. (2014). Can callous-unemotional traits enhance the understanding, diagnosis, and treatment of serious conduct problems in children and adolescents? A comprehensive review. Psychological Bulletin, 140, 1-57.

Jackson, D. (1989). Personality research form manual (3rd edn). New York: Research Psychologists.

Kochanska, G. (1991). Patterns of inhibition to the unfamiliar in children of normal and affectively ill mothers. Child Development, 62, 250-263.

Kochanska, G. (1997). Multiple pathways to conscience for children with different temperaments: From toddlerhood to age 5. Developmental Psychology, 33, 228-240.

Lahey, B.B., Rathouz, P.J., Applegate, B., Tackett, J.L., \& Waldman, I.D. (2010). Psychometrics of a self-report version of the Child and Adolescent Dispositions Scale. Journal of Clinical Child and Adolescent Psychology, 39, 351-361.

Mills-Koonce, W.R., Willoughby, M.T., Garrett-Peters, P., Wagner, N., \& Vernon-Feagans, L. (2016). The interplay among socioeconomic status, household chaos, and parenting in the prediction of child conduct problems and callousunemotional behaviors. Development and Psychopathology, 28, 757-771.

Muthén, L.K., \& Muthén, B.O. (2014). Mplus user's guide (7th edn) (1998-2014). Los Angeles: Muthén and Muthén.

Pasalich, D.S., Dadds, M.R., Hawes, D.J., \& Brennan, J. (2011). Do callous unemotional traits moderate the relative importance of parental coercion versus warmth in child conduct problems? An observational study. Journal of Child Psychology and Psychiatry, 52, 1308-1315.

Scott, S., Knapp, M., Henderson, J., \& Maughan, B. (2001). Financial cost of social exclusion: Follow up study of antisocial children into adulthood. British Medical Journal, 323, 191-194.

Shaw, D.S., Gilliom, M., Ingoldsby, E.M., \& Nagin, D.S. (2003). Trajectories leading to school-age conduct problems. Developmental Psychology, 39, 189-200.

Vanderbilt-Adriance, E., \& Shaw, D.S. (2008). Protective factors and the development of resilience in the context of neighborhood disadvantage. Journal of Abnormal Child Psychology, 36, 887-901.

Viding, E., Fontaine, N.M., \& McCrory, E.J. (2012). Antisocial behaviour in children with and without callous-unemotional traits. Journal of the Royal Society of Medicine, 105, 195-200.

Waller, R., Dishion, T.J., Shaw, D.S., Gardner, F., Wilson, M., \& Hyde, L.W. (2016). Does early childhood callous-unemotional behavior uniquely predict behavior problems or callous-unemotional behavior in late childhood? Developmental Psychology, 52, 1805-1819.

Waller, R., Gardner, F., \& Hyde, L.W. (2013). What are the associations between parenting, callous-unemotional traits, and antisocial behavior in youth? A systematic review of evidence. Clinical Psychology Review, 33, 593-608.

Waller, R., Gardner, F., Viding, E., Shaw, D.S., Dishion, T.J., Wilson, M.N., \& Hyde, L.W. (2014). Bidirectional associations between parental warmth, callous unemotional behavior, and behavior problems in high-risk preschoolers. Journal of Abnormal Child Psychology, 42, 1275-1285.

Waller, R., Hyde, L.W., Grabell, A.S., Alves, M.L., \& Olson, S.L. (2015). Differential associations of early callous unemotional, oppositional, and ADHD behaviors: Multiple domains within early starting conduct problems? Journal of Child Psychology and Psychiatry, 56, 657-666.

Waller, R., Shaw, D.S., Forbes, E.E., \& Hyde, L.W. (2015). Understanding early contextual and parental risk factors for the development of limited prosocial emotions. Journal of Abnormal Child Psychology, 43, 1025-1039.

Waller, R., Shaw, D.S., Neiderhiser, J.M., Ganiban, J.M., Natsuaki, M.N., Reiss, D., .. \& \& Hyde, L.W. (2017). Towards an understanding of the role of the environment in the development of early callous behavior. Journal of Personality, 85, 90-103.

Waller, R., Trentacosta, C.J., Shaw, D.S., Neiderhiser, J.M., Ganiban, J.M., Reiss, D., .. \& \& Hyde, L.W. (2016). Heritable temperament pathways to early callous-unemotional behavior. British Journal of Psychiatry, 209, 475-482.

Willoughby, M.T., Mills-Koonce, R.W., Gottfredson, N.C., \& Wagner, N.J. (2014). Measuring callous unemotional behaviors in early childhood: Factor structure and the prediction of stable aggression in middle childhood. Journal of Psychopathology and Behavioral Assessment, 36, 30-42.

Willoughby, M.T., Waschbusch, D.A., Moore, G.A., \& Propper, C.B. (2011). Using the ASEBA to screen for callous unemotional traits in early childhood: Factor structure, temporal stability, and utility. Journal of Psychopathology and Behavioral Assessment, 33, 19-30.

Winslow, E., \& Shaw, D. (1995). Early parenting coding system. Unpublished manuscript. University of Pittsburgh, Pittsburgh, PA, USA.

Accepted for publication: 19 October 2016

First published online: 5 December 2016 